\title{
Study on the Manipulation of Plasma Density around a Cubesat Using Magnetohydrodynamics
}

\author{
Filipe Dias ${ }^{1}$, Carlos Xisto ${ }^{2}$, and José Páscoa ${ }^{1}$ \\ ${ }^{1}$ Universidade da Beira Interior \\ ${ }^{2}$ Chalmers University of Technology
}

\section{Abstract}

During hypersonic flight regime or re-entry flights, a phenomenon known as radio blackout occurs, in which the high velocities attained lead to a significant increase in temperature surrounding the vehicle. This raise is so substantial that the molecules around the vehicle start to ionize, surrounding the vehicle in electrons. These electrons will prevent electromagnetic waves from leaving or reaching the aircraft, preventing all communications. Several blackout mitigation schemes have been proposed, among which is the magnetic window. In this scheme, a magnetic field is imposed near

Corresponding Author:

Filipe Dias

filipe.pinheiro.dias@ubi.pt

Received: 26 November 2019

Accepted: 13 May 2020

Published: 2 June 2020

Publishing services provided by Knowledge E

(c) Filipe Dias et al. This article is distributed under the terms of the Creative Commons

Attribution License, which permits unrestricted use and redistribution provided that the original author and source are credited.

Selection and Peer-review under the responsibility of the ICEUBI2019 Conference Committee.

\section{G OPEN ACCESS} the nose of the aircraft, which will then prevent electron movement, generating a spectral window through which the electromagnetic waves can pass. In this work, the effectiveness of this method in affecting the plasma density is tested. The mesh is tested for grid independency, ensuring an accurate solution in a sensible ammount of time. The effect of different magnetic field intensities is then tested for a CubeSat flying in hypersonic flight regime, in order to determine the effect of the magnetic field in the electron number density.

\section{Keywords: Magnetohydrodynamics, Radio blackout, Magnetic window, CubeSat}

\section{Introduction}

When a vehicle achieves hypersonic flight regime, for example, in an atmospheric reentry scenario, the collisions with the air molecules will steeply increase the temperature around the vehicle. The high temperatures will enable ionization reactions among the molecules, enveloping the vehicle in cations and electrons. Electromagnetic waves that try to reach or leave the vehicle are reflected and refracted by the electron layer, corrupting the transmitted data. This severely hampers the success of the mission, since the vehicle has no access to real time telemetry, GPS signal or radio to and from ground control. This phenomenon is known as radio blackout, and there has been an increasing interest in developing schemes for its mitigation [1]. 
One of those schemes is the magnetic window, in which a magnetic field is generated in the plasma sheath. The magnetic field will prevent electron movement, significantly decreasing local plasma density, which creates a passage whereby the electromagnetic waves can pass without being attenuated. This method can be considered either as a passive or an active method. If permanent magnets are used, it is considered a passive method. If electromagnets are used, it is possible to control the magnetic field intensity, marking this as an active method [2].

Russo and Hughes [3] performed experimental tests, determining that a magnetic field of $0.75 \mathrm{~T}$ is sufficient to mitigate radio blackout, showing that the magnetic window method is capable of mitigating radio blackout. However, the magnetic window presents some disadvantages, the main being the considerable weight of magnets, which increases fuel costs to prohibitive levels [4].

The employment of electromagnets also allows the usage of a time varying magnetic field, as is proposed by Stenzel and Urrutia [5]. The variable electric field will magnetize the electrons, expelling them from the field, which creates a Hall electronic field, expelling the ions. The resulting magnetic window allows the passage of the electromagnetic waves unhindered.

Experimental studies of hypersonic flight regimes are exceptionally expensive, due to the extreme conditions to which the aircraft is subject. As such, there is significant interest in hypersonic numerical simulations, which provide cheaper and more malleable solutions. Numerical simulations are often used in parametric studies, as nearly all parameters can be quickly altered, at no additional cost. This study is often followed by the construction of a prototype that will be used to confirm the numerical results.

In 2014 Xisto et. al [6] developed a code in which that solved resistive MHD equations, which was then validated with recourse to several well-known MHD test cases. The authors concluded that the proposed model could accurately simulate several different flow conditions. In 2015 Xisto et. al [7] used MHD principles in order to accelerate plasma in a MPD thruster. In 2016 Dias et. al [2] studied the effect of the orientation and intensity of the magnetic dipole on the flow around the RAM-C spacecraft flying in hypersonic regime, concluding that a horizontal dipole could affect the shockwave standoff distance more than a vertical dipole.

In this paper we will study the effectiveness of the magnetic window method in affecting the plasma density. A grid independence study is performed, ensuring an accurate solution for the lowest possible computational time. The effect of different magnetic field intensities on a CubeSat in hypersonic regime is then studied, determining the range of control over the plasma density. 
The code used in this study was developed in the open source software OpenFOAM.

\section{Governing Equations}

Previous works [2] have studied the effect of the magnetic window method on shockwave position, using a perfect gas model. A perfect gas model, however, does not consider several phenomena that occur in hypersonic flight regime, such as chemistry and thermal non- equilibrium, significantly lowering the code accuracy [8]. As such, a real gas model must be used, such as the one provided in [9],

The numerical code that was used in this study consists on the Navier Stokes equations, considering 11 species: $\mathrm{N}_{2}, \mathrm{O}_{2}, \mathrm{NO}, \mathrm{N}, \mathrm{O}, \mathrm{N}^{+}, \mathrm{O}_{2}{ }^{+}, \mathrm{NO}^{+}, \mathrm{N}^{+}, \mathrm{O}^{+}$and $\mathrm{e}^{-}$:

$$
\begin{gathered}
\frac{\partial \rho}{\partial t}+\nabla \cdot \rho \overrightarrow{\boldsymbol{U}}=0 \\
\frac{\partial \rho_{s}}{\partial t}+\nabla \cdot \rho_{s} \overrightarrow{\boldsymbol{U}}-\nabla \cdot\left(-\overrightarrow{\boldsymbol{J}}_{s}\right)=\dot{\omega}_{s} ; \\
\frac{\partial \rho \overrightarrow{\boldsymbol{U}}}{\partial t}+\nabla \cdot(\rho \overrightarrow{\boldsymbol{U}} \overrightarrow{\boldsymbol{U}}+p)-\nabla \cdot \tau=\overrightarrow{\boldsymbol{J}} \times \overrightarrow{\boldsymbol{B}} \\
\frac{\partial E}{\partial t}+\nabla \cdot\left((E+p) \overrightarrow{\boldsymbol{U}}-\tau \overrightarrow{\boldsymbol{U}}+\left(\overrightarrow{\boldsymbol{q}}_{v e}+\overrightarrow{\boldsymbol{q}}_{t r}\right)+\sum\left(\overrightarrow{\boldsymbol{J}}_{s} h_{s}\right)\right)=\mathbf{0}
\end{gathered}
$$

In which Eq. 1 is the mixture continuity equation, Eq. 2 is the species continuity equation, Eq. 3 is the momentum conservation equation and Eq. 4 is the energy conservation equation.

In this system of equations, $\rho$ and $\rho_{s}$ are, respectively, the mixture and species density; $\overrightarrow{\boldsymbol{U}}$ is the flow velocity; $\overrightarrow{\boldsymbol{J}}_{s}$ is the species mass diffusion flux; the continuity equation source term $\dot{\omega}_{s}$ is the species mass production rat; $p$ is the mixture pressure, calculated using Dalton's law; $\tau$ is the viscous term; $\overrightarrow{\boldsymbol{J}}$ and $\overrightarrow{\boldsymbol{B}}$ are respectively the electric current density and the imposed magnetic field; and $E$ is the total energy.

The species mass fraction is calculated by:

$$
Y_{s}=\frac{\rho_{s}}{\rho}
$$

The mass diffusion flux for each species can be calculated by a modified version of Fick's law, ensuring that the sum of all species mass diffusion flux is zero [10]:

$$
\overrightarrow{\boldsymbol{J}_{s \neq e}}=-\rho D \nabla Y_{s}-Y_{s} \sum_{r \neq e}-\rho D \nabla Y_{r}
$$


The mass production rate for each species is given by the expression:

$$
\dot{\omega}_{s}=M_{s} \sum_{k}\left(\beta_{s k}-\alpha_{s k}\right)\left[k_{f k} \prod_{j}\left(\frac{\rho_{j}}{M_{j}}\right)^{\alpha_{j k}}-k_{b k} \prod_{j}\left(\frac{\rho_{j}}{M_{j}}\right)^{\beta_{j k}}\right] ;
$$

Since the considered reactions are reversible, the production rate considers both forward and backward reaction rates. The forward reaction rate is given by the modified Arrhenius equation:

$$
k_{f k}=C_{f k} T^{\eta k} \exp \left(\frac{-\theta_{k}}{T}\right)
$$

Where the coefficients $C_{f k}, \eta k$ and $\theta_{k}$ are specific to the chemistry model used. In this study, the model proposed by Park was used, and the coefficients can be found in [11].

The plasma density is given by the electron number density:

$$
n_{e}=\frac{N_{A}}{M_{e}} \rho_{e}
$$

In which $N_{A}$ is the Avogadro constant. This expression will give the number of electrons per cubic meter, which will be the comparison term for the effects of the magnetic field on the flow.

The momentum equation source term is given by the Lorentz force, used to calculate the effect of the magnetic and electric field on the flow:

$$
\overrightarrow{\boldsymbol{J}}=\sigma(\overrightarrow{\boldsymbol{E}}+\overrightarrow{\boldsymbol{U}} \times \overrightarrow{\boldsymbol{B}})
$$

In which the electric field $\overrightarrow{\boldsymbol{E}}$ is considered equal to zero in this study. The pressure is given by Dalton's law of partial pressures of a gaseous mixture [12]:

$$
p=\sum \rho_{s} R_{s} T_{t r}
$$

The viscosity is given by the expression:

$$
\mu=\sum \frac{Y_{s} \mu_{s}}{\Phi_{s}}
$$

Where $\mu_{s}$ is the species viscosity, which can be calculated using Blottner's curve fits [13]:

$$
\mu_{s}=0.1 \exp \left[\left(A_{s} \ln T+B_{s}\right) \ln T+C_{s}\right]
$$

The energy per unit volume of the mixture is given by eq. 27 :

$$
E=\sum_{s \neq e} \rho_{s} C v_{t r, s} T_{t r}+\frac{1}{2} \rho\left(u^{2}+v^{2}+w^{2}\right)+\sum_{s \neq e} \rho_{s} h_{s}^{0}
$$

Where $h_{s}^{0}$ is the species enthalpy of formation at $0 \mathrm{~K}$, and $u, v$ and $w$ are the velocity components for each direction. 


\section{Mesh Refinement Study}

In computational fluid dynamics, the mesh employed plays a major role in the accuracy of the results. If a mesh is too coarse, the results will lose accuracy, especially if shockwaves are involved. If a mesh is too refined, however, the simulation runtime will increase to impractical levels. As such, a balance must be struck between accuracy and simulation runtime.

In this test case, a hypersonic flow over a CubeSat was calculated for three different meshes, as can be seen in Figure 1.
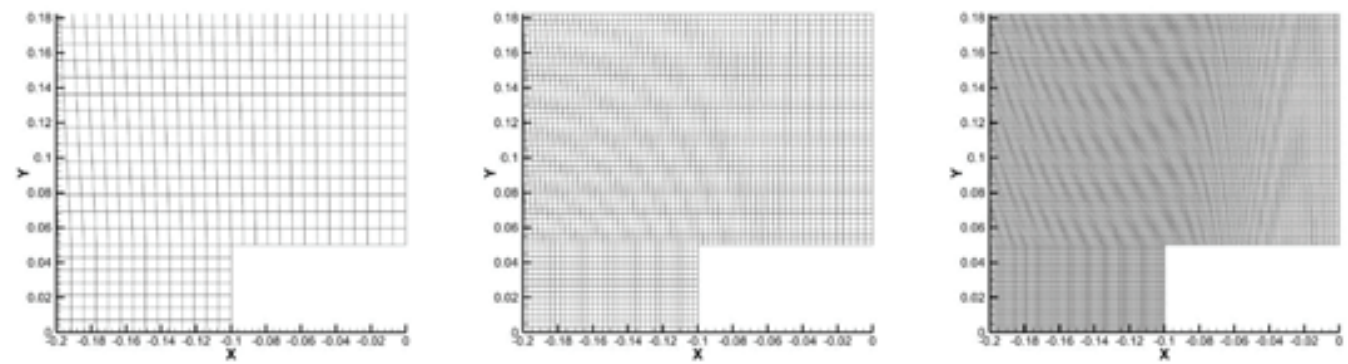

Figure 1: Meshes used in the test. Mesh 1 (left): 24200 points; Mesh 2 (center): 97600 points; Mesh 3 (right): 392000.

Regarding initial and boundary conditions: the flow is hypersonic with a Mach number of 24.7 , and a freestream velocity of $7669 \mathrm{~m} / \mathrm{s}$. Atmospheric pressure and temperature were set to $19 \mathrm{~Pa}$ and $240 \mathrm{~K}$. With those boundary conditions, the air density was $2.758 \mathrm{E}$ $4 \mathrm{~kg} / \mathrm{m}^{3}$. The freestream air was composed of $77 \% \mathrm{~N}_{2}$ and $23 \% \mathrm{O}_{2}$. Nine additional species were considered, namely: $\mathrm{NO}, \mathrm{N}, \mathrm{O}, \mathrm{N}_{2}^{+}, \mathrm{O}_{2}^{+}, \mathrm{NO}^{+}, \mathrm{N}^{+}, \mathrm{O}^{+}$and $\mathrm{e}^{-}$. These species, however, were not considered in the freestream, and can only be created via chemical reactions.



Figure 2: Pressure distribution in the shockwave region for different meshes 
The results obtained with the different meshes are shown in Fig.2. The plot portrays the pressure distribution in $y=0$, encompassing the shockwave region. It can be concluded that Mesh 3 presents the most accurate shockwave. The shockwaves calculated with Mesh 1 and Mesh 2 start very close to each other, but end far apart, with Mesh 1 being more dissipative than Mesh 2 .

The simulation runtime for Mesh 1 was 121 764s, for Mesh 2 was 72 742s and for Mesh 3 was 1009 370s. Despite having a quarter of the points, Mesh 1 takes twice as long to run as Mesh 2 because it required a Courant number of 0.1 to run without diverging, whilst Mesh 2 and Mesh 3 achieved convergence with a courant number of 0.9.

Due to the impractically long time needed to run a simulation with Mesh 3 and the high dissipation that occurred in Mesh 1, Mesh 2 was chosen for the remainder of the tests.

\section{Magnetohydrodynamic Manipulation of Plasma Density}

The CubeSat Launch Initiative (CSLI) is a NASA program that aims to provide a cheap and standardized launching platform for NASA missions, in which pre-existing rocket launches can transport and deploy CubeSats. A CubeSat is a nanosatellite consisting of one or more units with $10 \times 10 \times 11 \mathrm{~cm}$ each, generally weighing less than $1.4 \mathrm{~kg}$ per unit. The standard dimensions and usage of pre-planned launches have attracted the attention of several universities and private companies, providing their students and workers with a cheap and easy introduction to satellite development and space mission planning [14].

The initial and boundary conditions used in the mesh refinement study were used in this test as well, with the exception of the magnetic field. A dipole was applied to the coordinate $(x, y)=(-0.08 ; 0)$ as can be seen in figure 3 . Five different magnetic field intensities were applied: 0T; 0,25T; 0,5T; 0,75T; 1T. A magnetic dipole does not have a uniform magnetic field intensity, and as such, the tested values were taken in the CubeSat wall, where they were highest. Figure 3 shows the magnetic field intensity contours. Previous studies [2] found that a horizontal dipole was more effective in controlling the shockwave, and, as such, a horizontal dipole was used. The atmospheric electric conductivity was defined as $\sigma=200 \mathrm{~S} / \mathrm{m}$

Figures 4 and 5 represent, respectively, the electron number density and the pressure contours for all test cases.

It can be seen that, as the magnetic field intensity increases, the electron number density in the front of the CubeSat also increases, indicating an increase in the localized 



Figure 3: Magnetic field intensity for each case: Top left: 0,25T; Top right: 0.5T; Bottom left: 0.75T; Bottom right: $1 \mathrm{~T}$.

reaction rates. This increase in concentration can occur due to the orientation of the dipole. Both clockwise and counter clockwise horizontal dipole were studied, and similar results were obtained for both cases. A vertical dipole is being tested currently, as well as a uniform magnetic field, in order to determine if this behaviour occurs then as well.

As can be seen in Figure 5, the shockwave standoff distance is not affected by the magnetic field intensity, contrary to what was reported in [2]. This can occur due to a low electric conductivity. Future works will implement Spitzer's conductivity in the numerical code, and simulate the atmospheric conductivity. Another possible reason is the low spread and intensity of the magnetic field. Despite a maximum value of $1 \mathrm{~T}$ in one of the cases, the magnetic field is concentrated on the front of the CubeSat, well inside the shockwave, whilst the cited study had the magnetic field interacting with the shockwave. Future work will test higher magnetic field intensities and different locations, such as the corner of the CubeSat. 

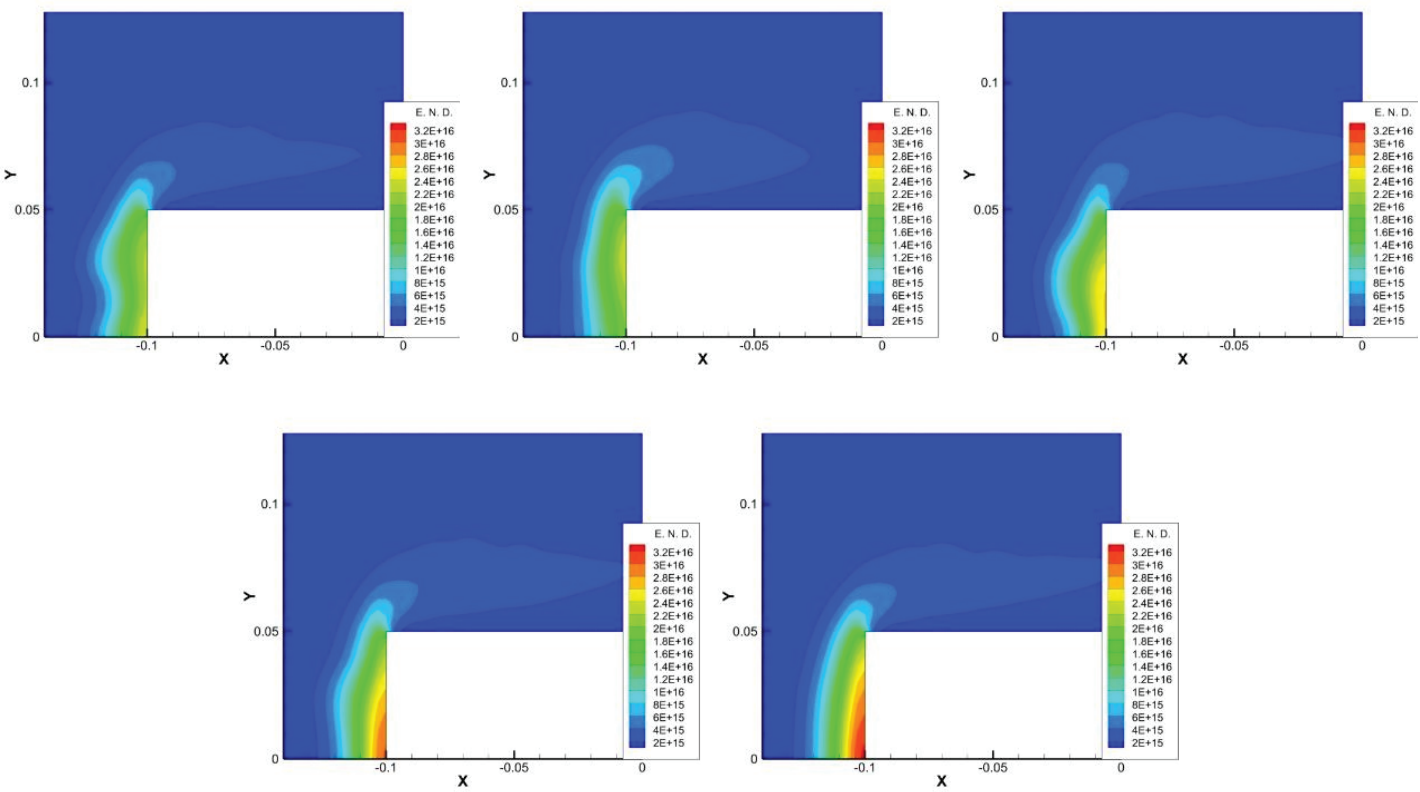

Figure 4: Electron Number Density for different magnetic field intensities. Top left: 0T; Top center: 0,25T; Top Right: 0,5T; Bottom Left: 0,75T; Bottom Right: 1T.
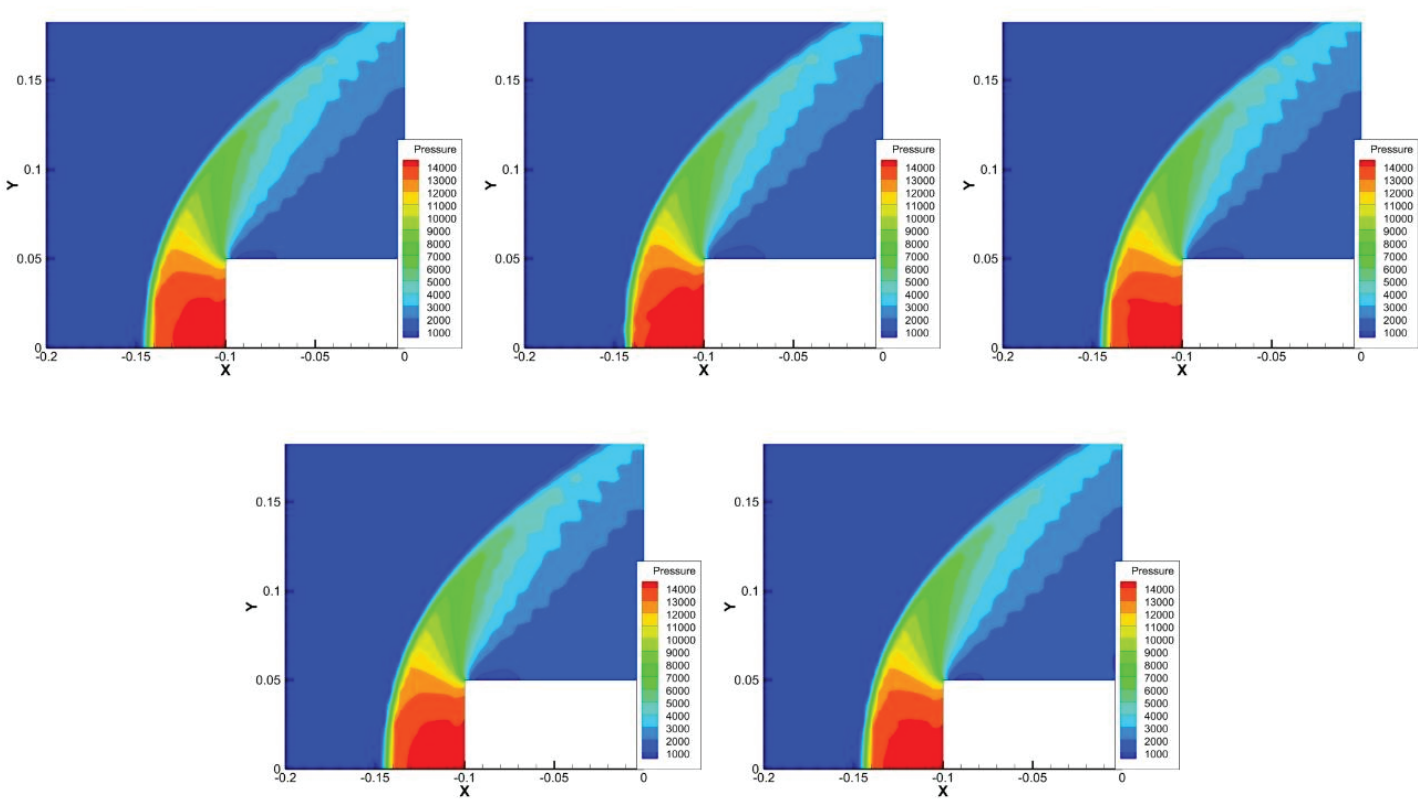

Figure 5: Pressure contours for different magnetic field intensities. Top left: 0T; Top center: 0,25T; Top Right: 0,5T; Bottom Left: 0,75T; Bottom Right: 1T.

\section{Conclusions}

In this work, the effectiveness of a well-known blackout mitigation method called magnetic window was tested. A grid independence study was performed, allowing the selection of a sufficiently accurate mesh that allowed a low simulation runtime. 
The effect of five different magnetic field intensities was tested on a CubeSat in hypersonic regime, showing that as the magnetic field intensity increased, so did the electron number density. It was also shown that the magnetic field did not affect the shockwave standoff distance, which might have occurred because the magnetic field was mainly concentrated inside the shockwave.

Future works will try to implement Spitzer's conductivity and perform additional tests, with different dipole positions and orientation.

\section{Acknowledgements}

The present work was supported by the Portuguese Foundation for Science and Technology (FCT), with the project nr. PTDC/SEM-TRA/5728/2014-POCl-01-0145-FEDER16662-HyperMHD "Magnetoplasmadynamic Flow Manipulation on Hypersonic and Reentry Vehicles". Additional financial support was provided by C-MAST - Center for Mechanical and Aerospace Sciences and Technologies, Research Unit No. 151.

\section{References}

[1] Kim, M. K., 2009. "Electromagnetic manipulation of plasma layer for re-entry blackout mitigation". Phd, University of Michigan.

[2] Dias, F., Xisto, C. M., and Pascoa, J. C., 2016. "Numerical computations of mhd flow on re- entry and hypersonic vehicles". In ASME 2016 International Mechanical Engineering Congress \& Exposition, Phoenix, Arizona, USA.

[3] Russo, F. P., and Hughes, J. K., 1964. "Measurements of The Effects of Static Magnetic Fields on VHF Transmission in Ionized Flow Fields." Tech. Rep. TM X-907, NASA.

[4] Hartunian, R. A., Stewart, G. E., Fergason, S. D., Curtiss, T. J., and Seibold, R. W., 2007. "Causes and Mitigation of Radio Frequency ( RF ) Blackout During Reentry of Reusable Launch Vehicles". Tech. Rep. 5309, The Aerospace Corporation.

[5] Stenzel, R. L., and Urrutia, J. M., 2013. "A New Method for Removing the Blackout Problem on Reentry Vehicles". Journal of Applied Physics, 113(10), pp. 1-5.

[6] Xisto, C. M., Pascoa, J. C., and Oliveira, P. J., 2014. "A pressure-based high resolution numerical method for resistive mhd". Journal of Computational Physics, 275, pp. 323-345.

[7] Xisto, C. M., Pascoa, J. C., and Oliveira, P. J., 2015. "Numerical analysis of real gas mhd flow on two-dimensional self-field mpd thrusters". Acta Astronautica, 112, pp. 89-101. 
[8] Scalabrin, L. C., 2007. "Numerical Simulation of Weakly lonized Hypersonic Flow Over Reentry Capsules". PhD thesis, University of Michigan.

[9] Dias, F., Pascoa, J., and Xisto, C., 2017. "A multi-species real gas model for the simulation of plasma flow in re-entry vehicles". In International Congress on Engineering, Covilhã, Portugal.

[10] Sutton, K.; Gnoffo, P. A., 1998: "Multi-component diffusion with application to computational aerothermodynamics", AIAA Paper 1998-2575.

[11] Park, C., 1990. "Nonequilibrium Hypersonic Aerothermodynamics". WileyInterscience.

[12] Silberberg, Martin S., 2009: "Chemistry: the molecular nature of matter and change". McGrawHill.

[13] Blottner, F. G.; Johnson, M.; and Ellis, M., 1971: "Chemically reacting viscous flow program for multi-component gas mixtures". SC-RR-70-754, Sandia Laboratories, Albuquerque, New Mexico.

[14] Crusan, J.; Galica, C., 2016: “ NASA's CubeSat Launch Initiative: Enabling Broad Access to Space", In 67th International Astronautical Congress (IAC), Guadalajara, Mexico. 